\section{Closed-Form Formulae for Time-Difference-of-Arrival Estimation}

Hing Cheung So, Senior Member, IEEE, Yiu Tong Chan, Senior Member, IEEE, and Frankie Kit Wing Chan

\begin{abstract}
For a positioning system with $L$ sensors, a maximum of $L(L-1) / 2$ distinct time-difference-of-arrival (TDOA) measurements, which are referred to as the full TDOA set, can be obtained. In this paper, closed-form expressions regarding optimum conversion of the full TDOA set to the nonredundant TDOA set, which corresponds to $(L-1)$ TDOA measurements with respect to a common reference receiver, in the case of white signal source and noise, are derived. The most interesting finding is that optimum conversion can be achieved via the standard least squares estimation procedure. Furthermore, the Cramér-Rao lower bound for TDOA-based positioning is produced in closed-form, which will be useful for optimum sensor array design.
\end{abstract}

Index Terms-Optimum processing, source localization, time delay estimation.

\section{INTRODUCTION}

Estimation of the time differences of arrival (TDOAs) between noisy versions of the same signal received at spatially separated sensors has been an important research topic [1]-[3]. Classical applications for time delay estimation include source localization and speed sensing in sonar and radar systems. Recent requirements for TDOA-based positioning are speaker tracking by microphone arrays [4], third-generation mobile communication systems [5], wireless local area networks [6], as well as sensor networks [7]. For two-dimensional (2-D) positioning, each TDOA measurement, which is proportional to the differences in source-sensor range, defines a hyperbolic locus on which the source must lie and the position estimate can be obtained from the intersection of two or more hyperbolas.

For a positioning system with $L$ sensors, there are $L(L-1) / 2$ distinct TDOA measurements from all possible sensor pairs, and we call them the full TDOA set. Alternatively, the so-called spherical TDOA set [8], renamed the nonredundant set here, is a subset of the full set and contains only $(L-1)$ TDOAs measured from sensor pairs with a common reference sensor, can be utilized for localization. In fact, most of the TDOA-based positioning algorithms in the literature, such as [4] and [9]-[11], are based on the nonredundant set, while only a few [12], [13] have considered the full set. The motivation of this paper is to answer the fundamental question of whether the full or nonredundant TDOA sets can give optimum positioning performance. The results show that optimality can be attained by both sets if the nonredundant TDOA measurements are properly converted from the full set. This conclusion conflicts with [8], which did not consider optimum processing of the nonredundant set. The contributions here are as follows.

1) Optimum conversion of the full TDOA set to the nonredundant set, assuming that each sensor receives a white source signal in

Manuscript received October 12, 2006; revised October 12, 2007. The associate editor coordinating the review of this manuscript and approving it for publication was Dr. Zhi Tian. The work described in this paper was supported by a grant from the Research Grants Council of the Hong Kong Special Administrative Region, China (Project No. CityU 119606).

H. C. So and F. K. W. Chan are with Department of Electronic Engineering, City University of Hong Kong, Kowloon, Hong Kong (e-mail: hcso@ee.cityu. edu.hk; k.w.chan@student.cityu.edu.hk).

Y. T. Chan is with Department of Electrical and Computer Engineering, Royal Military College of Canada Kingston, ON K7K 7B4, Canada (e-mail: chan-yt@rmc.ca).

Digital Object Identifier 10.1109/TSP.2007.914342 white noise. Although the results agree with [14], we are able to produce the corresponding closed-form conversion expressions.

2) A proof that the Gauss-Markov nonredundant set estimates can be obtained from the full set with the use of the standard least squares (LS). The asymptotic cases of high and low signal-to-noise ratio (SNR) conditions have also been investigated.

3) A verification that for TDOA-based positioning, the full and optimized nonredundant sets give identical Cramér-Rao lower bound (CRLB), which demonstrates their efficiency.

Hence, localization algorithms should always use the nonredundant set, since there is a less number of equations to solve. In addition, the CRLB is in closed-form which will be beneficial for the design of optimum 2-D sensor arrays.

The rest of the paper is organized as follows. In Section II, we consider TDOA estimation for a white source signal in additive white noise. After optimally estimating the full TDOA set, the Gauss-Markov estimate of the nonredundant TDOA set is derived in closed form. It is shown that Gauss-Markov estimate is in fact equal to the standard LS estimate. The closed-form expression of the CRLB for TDOAbased positioning is given in Section III. Section IV provides numerical examples to validate the theoretical findings. Finally, conclusions are drawn in Section V.

\section{OPtimum CONVERSION OF Full SET to NONREDUNDANT SET}

The discrete-time signal received at the $i$ th sensor is

$$
z_{i}(n)=s\left(n-D_{i}\right)+q_{i}(n), \quad i=1,2, \cdots L, n=0,1, \cdots N-1
$$

where $s(n)$ is the passive source signal, and $q_{i}(n)$ and $D_{i} \in \mathbb{R}$ are the additive noise and signal propagation delay, respectively, at the $i$ th sensor. Without loss of generality, the sampling frequency is $1 \mathrm{~Hz}$. Prior to sampling, the continuous-time signals are first lowpass filtered with a cutoff frequency of $0.5 \mathrm{~Hz}$. That is, the discrete-time signals are obtained at the Nyquist rate, and they are band-limited between -0.5 and $0.5 \mathrm{~Hz}$. Following standard practices, $\left\{q_{i}(n)\right\}$ in (1) are zero-mean white Gaussian processes which are independent of $s(n)$. The derivation below assumes that $s(n)$ is also a zero-mean white Gaussian process. This means that the continuous-time source signal has a flat spectrum over the lowpass filter bandwidth. Then after sampling at the Nyquist rate, all samples in $s(n)$ are independent and identically distributed. Certainly not all sources have flat spectra, but many signals do possess approximately this property. Examples are in the localization of an explosion underground, in the air or underwater. In electronic warfare, localizing a wideband jammer or a direct sequence spread spectrum transmitter will also have $s(n)$ that are near white noise processes. The task of TDOA estimation is to find the time difference between $\left\{D_{i}\right\}$ from the $L \times N$ measurements of $\left\{z_{i}(n)\right\}$.

Let $D_{i, j}=D_{i}-D_{j}$, where $i>j$, be the TDOA between $z_{i}(n)$ and $z_{j}(n)$, which is generally not an integral multiple of the sampling interval. A full set of $\left\{D_{i, j}\right\}$ contains $L(L-1) / 2$ elements. It is well known [15] that for continuous-time white Gaussian signal and noises, the maximum-likelihood estimate is given by the cross-correlator peak. For the discrete-time signals of (1), the estimate of $D_{i, j}$ based on crosscorrelation, denoted by $\hat{D}_{i, j}$, is computed as

$$
\hat{D}_{i, j}=\arg \max _{\tilde{D}_{i, j}}\left\{r_{i, j}\left(\tilde{D}_{i, j}\right)\right\}
$$

where

$$
r_{i, j}\left(\tilde{D}_{i, j}\right)=\sum_{n=0}^{N-1} z_{i}(n) z_{j}\left(n-\tilde{D}_{i, j}\right)
$$


is the cross-correlation function of $z_{i}(n)$ and $z_{j}(n)$ with lag $\tilde{D}_{i, j}$, which denotes the variable for $D_{i, j}$, and thus its value is not necessarily an integer. It is worthy to mention that since we do not assume $\tilde{D}_{i, j} \in \mathbb{Z}$, the results derived in this paper are not immediately applicable for typical discrete-time TDOA estimation where $\hat{D}_{i, j}$ is restricted to be an integral multiple of the sampling interval. Utilizing the inverse discrete-time Fourier transform for $e^{-j \omega \tilde{D}_{i, j}}$, the time-shifted signal $z_{j}\left(n-\tilde{D}_{i, j}\right)$ is generated as [16]

$$
z_{j}\left(n-\tilde{D}_{i, j}\right)=\sum_{i=-P}^{P} z_{j}(n-i) \operatorname{sinc}\left(i-\tilde{D}_{i, j}\right)
$$

where $\operatorname{sinc}(v)=\sin (\pi v) /(\pi v)$ is the sinc function and $P$, whose ideal value is infinity, should be chosen large enough to make the modeling error negligible. Let $\sigma_{s}^{2}$ and $\left\{\sigma_{q_{i}}^{2}\right\}$ be the variances of $s(n)$ and $\left\{q_{i}(n)\right\}$, respectively, and their values are assumed known. Consider that $N$ is sufficiently large and $\mathrm{SNR}=\sigma_{s}^{2} / \sigma_{q}^{2}$ is identical at each sensor with $\sigma_{q}^{2}=\sigma_{q i}^{2}, i=1,2, \cdots, L$. When $\hat{D}_{i, j} \approx D_{i, j}$, the variance of $\hat{D}_{i, j}$ at $N \rightarrow \infty$, denoted by $\operatorname{var}\left(\hat{D}_{i, j}\right)$, is derived using a first-order approximation as (see Appendix A)

$$
\operatorname{var}\left(\hat{D}_{i, j}\right) \approx \frac{3(1+2 \mathrm{SNR})}{\pi^{2} \mathrm{NSNR}^{2}}
$$

Because of the optimality of the cross-correlator for continuous-time white signals, there is no surprise that $\operatorname{var}\left(\hat{D}_{i, j}\right)$ equals the CRLB for TDOA estimation [17] between two discrete-time sensor outputs, denoted by $\mathrm{CRLB}_{2}$. It is noteworthy to point out that an asymptotic variance expression for cross-correlation-based time-delay estimate has been derived in [18]. Apart from different derivation approaches, the variance of [18] is larger than $\mathrm{CRLB}_{2}$ mainly because suboptimal parabolic interpolation is used while we have employed the optimum sinc interpolator.

The cross covariance of $\hat{D}_{i, j}$ and $\hat{D}_{k, l}$ at $N \rightarrow \infty$, denoted by $\operatorname{cov}\left(\hat{D}_{i, j}, \hat{D}_{k, l}\right)$ is (see Appendix A)

$$
\begin{array}{r}
\operatorname{cov}\left(\hat{D}_{i, j}, \hat{D}_{k, l}\right) \\
\quad \approx \begin{cases}\frac{3}{\pi^{2} N S N R}, & i=k \text { and } j \neq l \text { or } i \neq k \text { and } j=l \\
-\frac{3}{\pi^{2} N S N R}, & i=l \text { and } j \neq k \text { or } i \neq l \text { and } j=k \\
0, & i \neq j \neq k \neq l .\end{cases}
\end{array}
$$

Note that in Appendix A, we have in fact derived the general forms of $\operatorname{var}\left(\hat{D}_{i, j}\right)$ and $\operatorname{cov}\left(\hat{D}_{i, j}, \hat{D}_{k, l}\right)$ when the SNR at each sensor is distinct. Let $\mathbf{D}_{\mathbf{f}}=\left[D_{2,1}, D_{3,1}, \cdots, D_{L, 1}, D_{3,2}, \cdots, D_{L, L-1}\right]^{T} \in$ $\mathbb{R}^{L(L-1) / 2 \times 1}$, where $T$ denotes transpose, be the full set TDOA vector and its estimate using cross correlation be $\hat{\mathbf{D}}_{\mathbf{f}}=\left[\hat{D}_{2,1}, \hat{D}_{3,1}, \cdots, \hat{D}_{L, 1}, \hat{D}_{3,2}, \cdots, \hat{D}_{L, L-1}\right]^{T}$. With the use of (5) and (6), it can be shown that the asymptotic covariance matrix of $\hat{\mathbf{D}}_{\mathbf{f}}$, denoted by $\mathbf{C}_{\mathbf{f}} \in \mathbb{R}^{L(L-1) / 2 \times L(L-1) / 2}$, is of the form

$$
\mathbf{C}_{\mathbf{f}}=\frac{3}{\pi^{2} N \mathrm{SNR}}\left(\mathbf{J} \mathbf{J}^{T}+\frac{1}{\mathrm{SNR}} \mathbf{I}_{\frac{L(L-1)}{2}}\right)
$$

where

$$
\mathbf{J}=\left[\begin{array}{llll}
\mathbf{J}_{1}^{T} & \mathbf{J}_{2}^{T} & \cdots & \mathbf{J}_{L-1}^{T}
\end{array}\right]^{T} \in \mathbb{R}^{\frac{L(L-1)}{2} \times L}
$$

with

$$
\mathbf{J}_{i}=\left[\begin{array}{lll}
\mathbf{0}_{(L-i) \times(i-1)} & -\mathbf{1}_{L-i} & \mathbf{I}_{L-i}
\end{array}\right] \in \mathbb{R}^{(L-i) \times L}
$$

and $\mathbf{I}_{i}, \mathbf{0}_{i \times j}$ and $\mathbf{1}_{i}$ represent the $i \times i$ identity matrix, $i \times j$ zero matrix and $i \times 1$ vector with all elements 1 , respectively. It is noteworthy that (7) agrees with the covariance matrix for the correlator scheme measurement error vector given in [14] but we are able to produce it in closed form.

In the noise-free case, the nonredundant TDOA set can generate the full TDOA set without errors, that is, the full set contains redundant TDOA measurements. Without loss of generality, we take the first sensor as the reference sensor and denote the nonredundant TDOA set as $\mathbf{D}_{\mathbf{s}}=\left[D_{2,1}, D_{3,1}, \cdots, D_{L, 1}\right]^{T} \in \mathbb{R}^{(L-1) \times 1}$. The full and nonredundant TDOA sets are related by

$$
\mathbf{D}_{\mathbf{f}}=\mathbf{H D}_{\mathbf{s}}
$$

where $\mathbf{H}=\mathbf{J K} \in \mathbb{R}^{L(L-1) / 2} \times L$ with $\mathbf{K}=\left[\mathbf{0}_{(L-1) \times 1} \mathbf{I}_{L-1}\right]^{T} \in$ $\mathbb{R}^{L \times(L-1)}$. Let $\hat{\mathbf{D}}_{\mathbf{s}}=\left[\hat{\hat{D}}_{2,1}, \hat{\hat{D}}_{3,1}, \cdots, \hat{\hat{D}}_{L, 1}\right]^{T}$ be the Gauss-Markov estimate of $\mathbf{D}_{\mathbf{s}}$ based on $\hat{\mathbf{D}}_{\mathbf{f}}$. Employing the inverse of $\mathbf{C}_{\mathbf{f}}$ as the weighting matrix, $\hat{\hat{D}}_{\mathbf{s}}$ is easily obtained as the weighted LS estimate

$$
\hat{\hat{\mathbf{D}}}_{\mathbf{s}}=\left(\mathbf{H}^{T} \mathbf{C}_{\mathbf{f}}^{-1} \mathbf{H}\right)^{-1} \mathbf{H}^{T} \mathbf{C}_{\mathbf{f}}^{-1} \hat{\mathbf{D}}_{\mathbf{f}}
$$

where $^{-1}$ denotes the matrix inverse. We have found the closed-form expression of $\mathbf{C}_{\mathbf{f}}^{-1}$ as

$$
\mathbf{C}_{\mathbf{f}}^{-1}=-\frac{\pi^{2} N \mathrm{SNR}^{3}}{3(1+L \mathrm{SNR})}\left(\mathbf{J} \mathbf{J}^{T}-\frac{1+L \mathrm{SNR}}{\mathrm{SNR}} \mathbf{I}_{\frac{L(L-1)}{2}}\right) .
$$

With the use of (11), $\mathbf{H}^{T} \mathbf{C}_{\mathbf{f}}^{-1}$ can be simplified to (see Appendix B)

$$
\mathbf{H}^{T} \mathbf{C}_{\mathbf{f}}^{-1}=\frac{\pi^{2} N \mathrm{SNR}^{2}}{3(1+L \mathrm{SNR})} \mathbf{H}^{T}
$$

so that

$$
\left(\mathbf{H}^{T} \mathbf{C}_{\mathbf{f}}^{-1} \mathbf{H}\right)^{-1} \mathbf{H}^{T} \mathbf{C}_{\mathbf{f}}^{-1}=\left(\mathbf{H}^{T} \mathbf{H}\right)^{-1} \mathbf{H}^{T} .
$$

Thus, the Gauss-Markov estimate of $\mathbf{D}_{\mathbf{s}}$ is in fact the standard LS estimate and computational complexity can be significantly reduced for optimum conversion of the full TDOA set to the nonredundant. Furthermore, the closed-form expression of $\left(\mathbf{H}^{T} \mathbf{H}\right)^{-1} \mathbf{H}^{T}$ is (see Appendix C)

$$
\left(\mathbf{H}^{T} \mathbf{H}\right)^{-1} \mathbf{H}^{T}=\frac{1}{L}\left[\mathbf{I}_{L-1}+\mathbf{1}_{L-1} \mathbf{1}_{L-1}^{T} \breve{\mathbf{H}}^{T}\right]
$$

where $\breve{\mathbf{H}}^{T}$ is a partition of $\mathbf{H}^{T}$ which contains only its last $(L-1)(L-$ 2) $/ 2$ columns. For reference sensors other than the first, we only need to change $\mathbf{H}^{T}$ accordingly to obtain the corresponding estimate of the nonredundant set. In fact, it can also be deduced easily from $\hat{\hat{\mathbf{D}}}_{\mathrm{s}}$ that the LS estimation procedure implies $\hat{\hat{D}}_{i, j}+\hat{\hat{D}}_{j, k}+\hat{\hat{D}}_{k, i}=0$ [13] That is, $\hat{\hat{D}}_{i, j}=\hat{\hat{D}}_{i, 1}-\hat{\hat{D}}_{j, 1}$ for $i \neq j \neq 1$. Note that the zero residual of $\left\{\hat{\hat{D}}_{i, j}\right\}$ is also mentioned in [19] for consistency checks but no suggestion is provided for achieving it.

Furthermore, the covariance matrix of $\hat{\hat{\mathbf{D}}}_{\mathbf{s}}$, denoted by $\mathbf{C}_{\mathbf{s}} \in$ $\mathbb{R}^{(L-1) \times(L-1)}$, is then

$$
\mathbf{C}_{\mathbf{s}}=\left(\mathbf{H}^{T} \mathbf{C}_{\mathbf{f}}^{-1} \mathbf{H}\right)^{-1} .
$$

With the use of (12), the closed-form expression for $\mathbf{C}_{\mathbf{s}}$ is (see Appendix C)

$$
\begin{aligned}
\mathbf{C}_{\mathbf{s}} & =\frac{3(1+L \mathrm{SNR})}{\pi^{2} N \mathrm{SNR}^{2}}\left(\mathbf{H}^{T} \mathbf{H}\right)^{-1} \\
& =\frac{3\left(1+L \mathrm{SNR}^{-1}\right.}{\pi^{2} L N \mathrm{SNR}^{2}}\left(\mathbf{I}_{L-1}+\mathbf{1}_{L-1} \mathbf{1}_{L-1}^{T}\right)
\end{aligned}
$$

while its inverse is (see Appendix C)

$$
\mathbf{C}_{\mathbf{s}}^{-1}=\frac{\pi^{2} \operatorname{NSNR}^{2}\left(L \mathbf{I}_{L-1}-\mathbf{1}_{L-1} \mathbf{1}_{L-1}^{T}\right)}{3(1+L \mathrm{SNR})} .
$$


Note that (17) will be employed for computing the CRLB for positioning using the estimate of the nonredundant TDOA set in the next section.

Recall that the Fisher information matrix (FIM) for TDOA estimates using the nonredundant set is [14]

$$
\begin{aligned}
\mathbf{F}=\frac{N}{2 \pi} \int_{-\pi}^{\pi} \omega^{2} \frac{S^{2}(\omega)}{1+\sum_{i=1}^{L} S(\omega) / Q_{i}(\omega)} \\
\quad \times\left[\operatorname{tr}\left(\mathbf{Q}^{-1}(\omega)\right) \mathbf{Q}_{p}^{-1}(\omega)-\mathbf{Q}_{p}^{-1}(\omega) \mathbf{1}_{L-1} \mathbf{1}_{L-1}^{T} \mathbf{Q}_{p}^{-1}(\omega)\right] d \omega
\end{aligned}
$$

where $S(\omega)$ and $Q_{i}(\omega)$ represent the power spectra of $s(k)$ and $q_{i}(k)$, respectively, while $\mathbf{Q}(\omega)=\operatorname{diag}\left(Q_{1}(\omega), Q_{2}(\omega), \cdots, Q_{L}(\omega)\right)$ and $\mathbf{Q}_{p}(\omega)=\operatorname{diag}\left(Q_{2}(\omega), Q_{3}(\omega), \cdots, Q_{L}(\omega)\right)$. It is noteworthy that

(18) is derived under the following assumptions:

1) the continuous-time random signals are wide-sense stationary processes;

2) instead of analyzing the signals in the time domain, they are analyzed in the frequency domain by sampling their Fourier transforms with frequency interval of $2 \pi / T$ where $T$ is the observation interval; since we have assumed unity sampling interval in (1), we have $T=N$;

3) the observation interval-bandwidth product of the processes is sufficiently large such that the Fourier samples are uncorrelated and thus the discrete summation can be changed to continuous integration.

For white signal and noises with variances $\sigma_{s}^{2}$ and $\sigma_{q}^{2}$, we have $S(\omega)=$ $\sigma_{s}^{2}, Q_{i}(\omega)=\sigma_{q}^{2}, i=1,2, \cdots, L, \mathbf{Q}(\omega)=\sigma_{q}^{2} \mathbf{I}_{L}$ and $\mathbf{Q}_{p}(\omega)=$ $\sigma_{q}^{2} \mathbf{I}_{L-1}$. Substituting these values into (18) with SNR $=\sigma_{s}^{2} / \sigma_{q}^{2}$, we can easily show that (17) and (18) are identical. Since the CRLB for $\mathbf{D}_{\mathbf{s}}$ is given by the inverse of the FIM, it follows that $\hat{\hat{\mathbf{D}}}_{\mathbf{s}}$ is the asymptotically optimum estimate of the nonredundant set. This finding is again consistent with [14], but we are able to produce the corresponding closed form expressions here. On the other hand, although Schmidt [13] has also suggested to employ the LS technique or averaging for improving the full set TDOA estimates, there is no discussion regarding the optimality issue.

From the diagonal of (15), the CRLB for $\left\{D_{i, 1}\right\}, i=2,3, \cdots, L$, using $L$ sensors, denoted by $\mathrm{CRLB}_{L}$, is

$$
\mathrm{CRLB}_{L}=\frac{6(1+L \mathrm{SNR})}{\pi^{2} L N \mathrm{SNR}^{2}} .
$$

Clearly, (19) agrees with (5) when $L=2$. The performance improvement of using $\hat{\mathbf{D}}_{\mathbf{s}}$ over the truncated set from $\hat{\mathbf{D}}_{\mathbf{f}}$, namely $\left\{\hat{D}_{2,1}, \hat{D}_{3,1}, \cdots, \hat{D}_{L, 1}\right\}$, can be investigated from the ratio of $\mathrm{CRLB}_{L}$ to $\mathrm{CRLB}_{2}$ as follows:

$$
\frac{\mathrm{CRLB}_{L}}{\mathrm{CRLB}_{2}}=\frac{2(1+L \mathrm{SNR})}{L(1+2 \mathrm{SNR})}
$$

For SNR 》1, this ratio approaches 1 , which indicates that there is no improvement and $\hat{\hat{D}}_{i, 1} \approx \hat{D}_{i, 1}, i=2,3, \cdots, L$. As a result, estimating only $\left\{\hat{D}_{i, 1}\right\}$ is sufficient to achieve optimum processing while computations can be saved because estimation of the remaining $\left\{\hat{D}_{i, j}\right\}$ and the LS procedure for calculating the Gauss-Markov estimate are not required. It is noteworthy that this finding conforms with (7) as $\mathbf{C}_{\mathbf{f}}$ will become an ill-conditioned matrix at $\mathrm{SNR} \gg 1$ due to the rank deficiency of $\mathbf{J J}^{T}$. At $\mathrm{SNR} \ll 1, \mathrm{CRLB}_{L} / \mathrm{CRLB}_{2}$ approaches $2 / L$ which means that the enhancement only increases linearly with respect to the sensor number. Interestingly, we have also found that for sufficiently small SNR, the covariance matrix is $\mathbf{C}_{\mathbf{f}} \approx$
$3 /\left(\pi^{2} N \mathrm{SNR}^{2}\right) \mathbf{I}_{L(L-1) / 2}$. With this value of $\mathbf{C}_{\mathbf{f}},(13)$ can be obtained much simpler.

\section{Positioning Performance Bound}

Let $\left[\begin{array}{ll}x & y\end{array}\right]^{T}$ and $\left[x_{i} y_{i}\right]^{T}, i=1,2, \cdots, L$, be the unknown target position and known sensor positions, respectively, then their relationship with the TDOAs is

$$
v D_{i, j}=\sqrt{\left(x-x_{i}\right)^{2}+\left(y-y_{i}\right)^{2}}-\sqrt{\left(x-x_{j}\right)^{2}+\left(y-y_{j}\right)^{2}}
$$

where $v$ represents the signal propagation speed which is a known constant. The minimum achievable mean square position error (MSPE), denoted by $E\left\{(\hat{x}-x)^{2}+(\hat{y}-y)^{2}\right\}$, where $\hat{x}$ and $\hat{y}$ represent the unbiased estimates of $x$ and $y$ and $E$ is the expectation operator, is developed as follows. The $2 \times 2$ FIM for 2-D positioning with the parameter vector $\left[\begin{array}{ll}x & y\end{array}\right]^{T}$ using the nonredundant set, denoted by $\mathbf{F}_{\mathbf{s}}$, is given by [11]

$$
\mathbf{F}_{\mathbf{s}}=\frac{\mathbf{G}_{\mathbf{s}} \mathbf{C}_{\mathbf{s}}^{-1} \mathbf{G}_{\mathbf{s}}^{T}}{v^{2}}
$$

where

$$
\begin{aligned}
\mathbf{G}_{\mathbf{s}} & =\left[\begin{array}{lll}
\mathbf{g}_{21} & \mathbf{g}_{31} & \cdots \mathbf{g}_{L 1}
\end{array}\right] \in \mathbb{R}^{2 \times(L-1)} \\
\mathbf{g}_{i 1} & =\mathbf{g}_{i}-\mathbf{g}_{1} \\
\mathbf{g}_{i} & =\left[\begin{array}{l}
g_{x, i} \\
g_{y, i}
\end{array}\right]=\left[\begin{array}{l}
\frac{x-x_{i}}{\sqrt{\left(x-x_{i}\right)^{2}+\left(y-y_{i}\right)^{2}}} \\
\sqrt{\left(x-x_{i}\right)^{2}+\left(y-y_{i}\right)^{2}}
\end{array}\right] .
\end{aligned}
$$

In Appendix D, we have expressed $\mathbf{G}_{\mathbf{s}} \mathbf{C}_{\mathbf{s}}^{-1} \mathbf{G}_{\mathbf{s}}^{T}$ as

$$
\mathbf{G}_{s} \mathbf{C}_{s}^{-1} \mathbf{G}_{s}^{T}=\frac{\pi^{2} N \mathrm{SNR}^{2}}{3(1+L \mathrm{SNR})}\left(L \mathbf{G}^{T} \mathbf{G}-\mathbf{G}^{T} \mathbf{1}_{L} \mathbf{1}_{L}^{T} \mathbf{G}\right)
$$

where

$$
\mathbf{G}=\left[\begin{array}{llll}
g_{x, 1} & g_{x, 2} & \cdots & g_{x, L} \\
g_{y, 1} & g_{y, 2} & \cdots & g_{y, L}
\end{array}\right]^{T} .
$$

Substituting (23) into (22) and then taking the inverse, we can obtain the closed-form CRLB for $x$ and $y$ from its diagonal elements.

On the other hand, the FIM for 2-D positioning using the full TDOA set, denoted by $\mathbf{F}_{\mathbf{f}}$, is given by [20]

$$
\mathbf{F}_{\mathbf{f}}=\frac{\mathbf{G}_{\mathbf{f}} \mathbf{C}_{\mathbf{f}}^{-1} \mathbf{G}_{\mathbf{f}}^{T}}{v^{2}}
$$

where

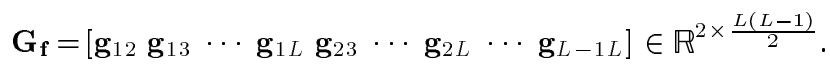

With the use of (15) and $\mathbf{G}_{\mathbf{f}}=\mathbf{G}_{\mathbf{s}} \mathbf{H}^{T}$, it is easy to obtain $\mathbf{G}_{\mathbf{f}} \mathbf{C}_{\mathbf{f}}^{-1} \mathbf{G}_{\mathbf{f}}^{T}=\mathbf{G}_{\mathbf{s}} \mathbf{C}_{\mathbf{s}}^{-1} \mathbf{G}_{\mathbf{s}}^{T}$. Hence, localization using the full or nonredundant sets will have the same CRLB. This is not a surprising result in view of the development in Section II. Hence, localization algorithms should always use the nonredundant set because there is a less number of equations to solve. The findings in this section can also contribute to the research of optimum 2-D sensor array design. Although Yang and Scheuing [8], [20] have performed some pioneering study on the impact of the sensor array geometry on the TDOA-based localization accuracy, their assumption of a scaled identity matrix for $\mathbf{C}_{\mathbf{f}}$ is rather restrictive. As seen in Section II, this is valid only for SNR $\ll 1$. In contrast, (23) provides the basis for study of sensor array geometry effects on the CRLB.

\section{Simulation STUdY}

Computer simulations have been performed to verify the theoretical development on TDOA-based positioning. A microphone array application scenario with eight sensors is considered and the speed of signal 


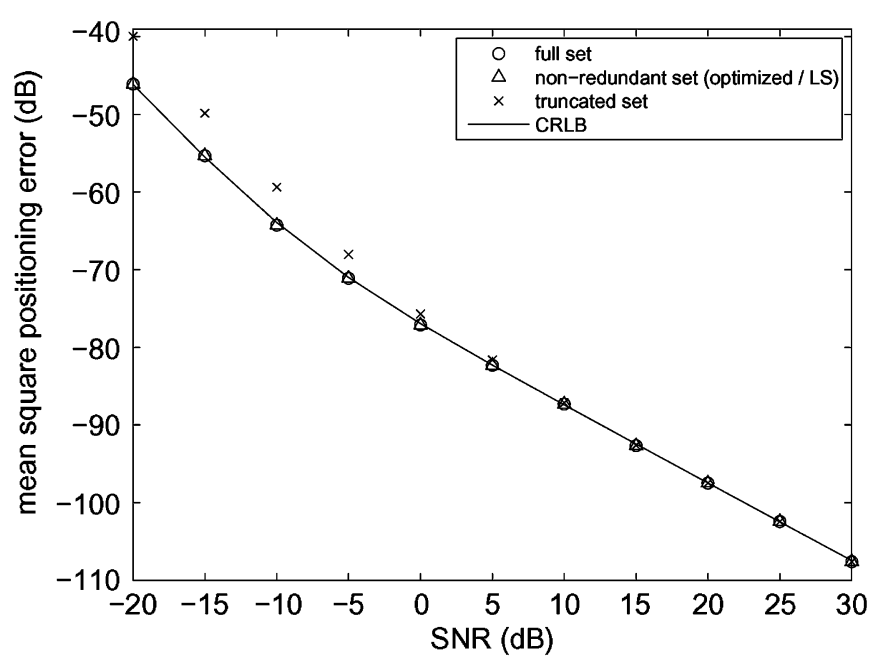

Fig. 1. Mean square position error when sensor outputs have same SNRs.

propagation is $v=340 \mathrm{~ms}^{-1}$. The eight microphones are at $(0,0) \mathrm{m}$, $(0,50) \mathrm{m},(0,100) \mathrm{m},(50,100) \mathrm{m},(100,100) \mathrm{m},(100,50) \mathrm{m},(100,0) \mathrm{m}$, and $(50,0) \mathrm{m}$, while the source position is at $(10,20) \mathrm{m}$. The MSPE is employed as the performance measure, and all results provided are averages of 1000 independent runs.

In the first test, the SNRs of all sensor outputs are identical. Based on the investigated geometry, the optimum full set TDOA estimates, namely, $\hat{\mathbf{D}}_{\mathbf{f}}$, are generated with the covariance matrix of (7) according to a Gaussian distribution. In doing so, we have implicitly assumed sufficiently high SNR conditions such that there are no large errors in the TDOA estimates. It should be emphasized that the TDOA estimation problem of (2) is nonlinear, and hence in practice, it will suffer from the threshold phenomenon when SNR is small enough. The optimum nonredundant estimate of $\mathbf{D}_{\mathbf{s}}$ is then obtained from $\hat{\mathbf{D}}_{\mathbf{f}}$ with the use of the LS procedure of (14). The performance of the truncated set $\left\{\hat{D}_{2,1}, \hat{D}_{3,1}, \cdots, \hat{D}_{8,1}\right\}$ is also evaluated. From each set of TDOA measurements, the position estimate, namely, $(\hat{x}, \hat{y})$, is determined by finding the maximum of the corresponding maximum likelihood cost functions. The Newton-Raphson iterative procedure is employed for the maximum search and the true position is selected as the initial guess. Again, we have ignored the threshold phenomenon. Fig. 1 shows the MSPEs of the three sets of TDOA estimates versus SNR. It is seen that the MSPEs based on the full set and optimized nonredundant set perform equally and meet the CRLB for all SNR conditions, which agrees with the findings in Section III. Furthermore, we observe that employing $\left\{\hat{D}_{2,1}, \hat{D}_{3,1}, \cdots, \hat{D}_{8,1}\right\}$ can provide optimum position estimates only at high SNRs, as predicted in Section II.

In the second test, we study the comparative positioning accuracy for different schemes of processing the TDOA measurements when the sensor outputs have unequal SNRs. We use the covariance matrix in Appendix A to generate the full set TDOA measurements. For simplicity, we set $\sigma_{q_{1}}^{2}=20 \sigma_{q}^{2}$ and $\sigma_{q}^{2}=\sigma_{q_{2}}^{2}=\sigma_{q_{3}}^{2}=\cdots=\sigma_{q_{L}}^{2}$ with $\mathrm{SNR}=\sigma_{s}^{2} / \sigma_{q}^{2}$, which implies that the truncated set $\left\{\hat{D}_{2,1}, \hat{D}_{3,1}, \cdots, \hat{D}_{8,1}\right\}$ is now more noisy than other TDOA estimates in the full set. The MSPEs of different methods are plotted in Fig. 2. Note that for this scenario, (13) does not hold and thus the results for the Gauss-Markov estimate of (10) are also included. Again, we observe that the estimators using the full set and optimized nonredundant set give optimum performance for the whole SNR range. Due to a smaller SNR condition at $\left\{\hat{D}_{2,1}, \hat{D}_{3,1}, \cdots, \hat{D}_{8,1}\right\}$, the MSPE from using the truncated set cannot meet the CRLB even at high SNRs. On the other hand, it is interesting to see that the performance of the simple LS procedure for TDOA's attains optimality

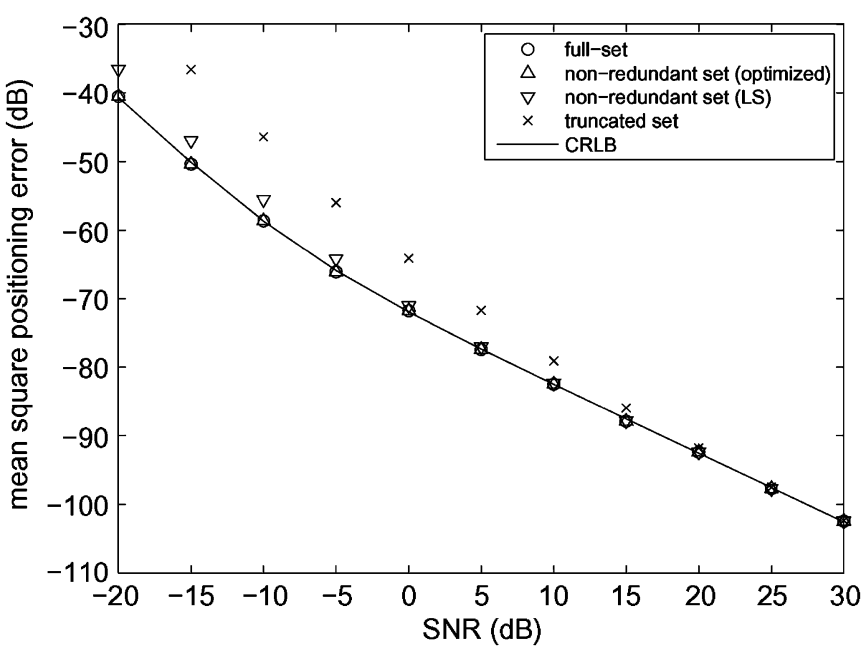

Fig. 2. Mean square position error when the first sensor output has smaller SNR.

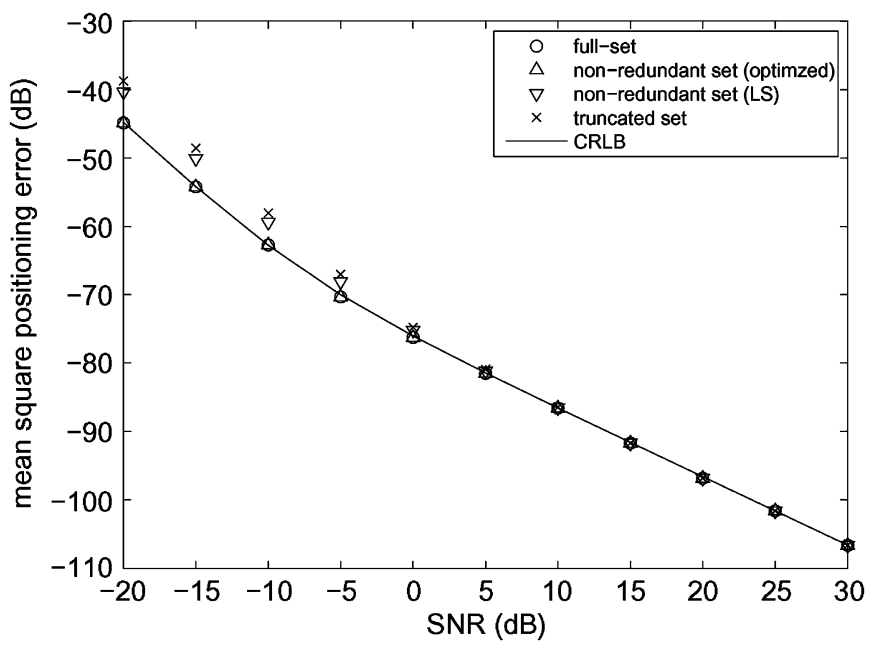

Fig. 3. Mean square position error when the second sensor output has smaller SNR.

for SNR $\in[-10,30] \mathrm{dB}$. This test is repeated with $\sigma_{q_{2}}^{2}=20 \sigma_{q}^{2}$ and $\sigma_{q}^{2}=\sigma_{q_{1}}^{2}=\sigma_{q_{3}}^{2}=\cdots=\sigma_{q_{L}}^{2}$, and the results are plotted in Fig. 3 . The observations are similar to those of Fig. 2 except that the truncated set can give optimum estimation performance at higher SNRs because now $\left\{\hat{D}_{2,1}, \hat{D}_{3,1}, \cdots, \hat{D}_{8,1}\right\}$ is generally less noisy compared to the remaining TDOA estimates in the full set.

\section{CONCLUSION}

Optimal conversion of the full time-difference-of-arrival (TDOA) set estimates to the nonredundant can be achieved using the standard least squares procedure and the corresponding closed form expressions are derived for the case of white signal in additive white noise. It is proved that both the full and nonredundant sets of TDOA estimates can give the same position estimation performance. Cramér-Rao lower bound for TDOA-based positioning is also derived in closed form, and this should facilitate the design of optimum sensor placement for localization.

\section{APPENDIX A}

The TDOA variance of the cross-correlator is first derived as follows. For simplicity but without loss of generality, we only consider TDOA estimation between $z_{1}(n)$ and $z_{2}(n)$ and let $D_{1}=0$ and $D=D_{2,1}=$ $D_{2}$ be the time difference. The powers of the Gaussian processes $s(k)$ 
and $q_{i}(k), i=1,2, \cdots, L$, are denoted by $\sigma_{s}^{2}$ and $\sigma_{q_{i}}^{2}$, respectively. The corresponding cross-correlator function is then

$$
r_{2,1}(\tilde{D})=\sum_{n=0}^{N-1} z_{2}(n) z_{1}(n-\tilde{D})
$$

where

$$
z_{1}(n-\tilde{D})=\sum_{i=-P}^{P} z_{1}(n-i) \operatorname{sinc}(i-\tilde{D}) .
$$

We consider the asymptotic condition of $N \rightarrow \infty$ and $P$ is chosen sufficiently large. Denote $\hat{D}$ as the TDOA estimate which is the $\tilde{D}$ which maximizes $r_{2,1}(\tilde{D})$. This implies

$$
\left.r_{2,1}^{\prime}(\tilde{D})\right|_{\tilde{D}=\hat{D}}=0
$$

where $r_{2,1}^{\prime}(\tilde{D})$ is the first derivative of $r_{2,1}(\tilde{D})$ with respect to $\tilde{D}$. When $E\left\{r_{2,1}^{\prime}(\tilde{D})\right\}$ is sufficiently smooth around $\tilde{D}=D$, we have the following first-order approximation [21]:

$$
\begin{aligned}
\left.\frac{d E\left\{r_{2,1}^{\prime}(\tilde{D})\right\}}{d \tilde{D}}\right|_{\tilde{D}=D} & =\left.E\left\{r_{2,1}^{\prime \prime}(\tilde{D})\right\}\right|_{\tilde{D}=D} \\
& \approx \frac{\left.r_{2,1}^{\prime}(\tilde{D})\right|_{\tilde{D}=\hat{D}}-\left.r_{2,1}^{\prime}(\tilde{D})\right|_{\tilde{D}=D}}{\hat{D}-D}
\end{aligned}
$$

where $r_{2,1}^{\prime \prime}(\tilde{D})$ is the corresponding second derivative. With the use of (A3) and (A4), the mean value of $\hat{D}$ is

$$
E\{\hat{D}\} \approx D-\left.\frac{E\left\{r_{2,1}^{\prime}(\tilde{D})\right\}}{E\left\{r_{2,1}^{\prime \prime}(\tilde{D})\right\}}\right|_{\tilde{D}=D}
$$

Employing (A1) and (A2), $\left.r_{2,1}^{\prime}(\tilde{D})\right|_{\tilde{D}=D}$ is

$$
\left.r_{2,1}^{\prime}(\tilde{D})\right|_{\tilde{D}=D}=-\sum_{n=0}^{N-1} \sum_{i=-P}^{P} z_{2}(n) z_{1}(n-i) \operatorname{sinc}^{\prime}(i-D) .
$$

For uncorrelated $q_{1}(n)$ and $q_{2}(n)$, taking the expected value of (A6) yields

$$
\begin{aligned}
E & \left.\left\{r_{2,1}^{\prime}(\tilde{D})\right\}\right|_{\tilde{D}=D} \\
= & -\sum_{n=0}^{N-1} \sum_{i=-P}^{P} E\{s(n-D) s(n-i)\} \operatorname{sinc}^{\prime}(i-D) \\
= & -\sum_{n=0}^{N-1} \sum_{i=-P}^{P} \sum_{j=-\infty}^{\infty} E\{s(n-i) s(n-j)\} \\
& \times \operatorname{sinc}^{\prime}(i-D) \operatorname{sinc}(j-D) \\
= & -N \sigma_{s}^{2} \sum_{i=-P}^{P} \operatorname{sinc} c^{\prime}(i-D) \operatorname{sinc}(i-D) \\
\approx & -N \sigma_{s}^{2} \operatorname{sinc}^{\prime}(0)=0
\end{aligned}
$$

because $\operatorname{sinc}^{\prime}(0)=0$. In a similar manner, the term $\left.E\left\{r_{2,1}^{\prime \prime}(\tilde{D})\right\}\right|_{\tilde{D}=D}$ is evaluated as

$$
\begin{aligned}
E & \left.\left\{r_{2,1}^{\prime \prime}(\tilde{D})\right\}\right|_{\tilde{D}=D} \\
& =\sum_{n=0}^{N-1} \sum_{i=-P}^{P} E\{s(n-D) s(n-i)\} \operatorname{sinc}^{\prime \prime}(i-D) \\
& \approx N \sigma_{s}^{2} \operatorname{sinc}^{\prime \prime}(0)=-\frac{N \sigma_{s}^{2} \pi^{2}}{3}
\end{aligned}
$$

with the use of $\operatorname{sinc} c^{\prime \prime}(0)=-\pi^{2} / 3$ [22]. Substituting (A7) and (A8) into (A5) yields

$$
E\{\hat{D}\} \approx D
$$

which shows the approximate unbiasedness of the cross-correlator.

Squaring both sides of (A4) with the use of (A3) and (A9), the variance of $\hat{D}$ based on the first-order approximation, is

$$
\begin{aligned}
\operatorname{var}(\hat{D})= & E\left\{(\hat{D}-E\{\hat{D}\})^{2}\right\} \\
& \approx E\left\{(\hat{D}-D)^{2}\right\} \\
& \left.\approx \frac{E\left\{r_{2,1}^{\prime 2}(\tilde{D})\right\}}{\left(E\left\{r_{2,1}^{\prime \prime}(\tilde{D})\right\}\right)^{2}}\right|_{\tilde{D}=D} .
\end{aligned}
$$

It is noteworthy that (A10) can also be derived by expanding $r_{2,1}^{\prime}(\tilde{D})$ in a Taylor series about $D$ and retaining only linear terms [23]. By squaring (A6), the numerator of (A10) is

$$
\begin{aligned}
E\{ & \left.r_{2,1}^{\prime 2}(D)\right\} \\
= & \sum_{n=0}^{N-1} \sum_{m=0}^{N-1} \sum_{i=-P}^{P} \sum_{j=-P}^{P} E\left\{z_{1}(n-i) z_{1}(m-j) z_{2}(n) z_{2}(m)\right\} \\
& \times \operatorname{sinc}^{\prime}(i-D) \operatorname{sinc}^{\prime}(j-D) .
\end{aligned}
$$

Using the properties that the signal and noise are uncorrelated and white Gaussian processes as well as the sinc function, namely, $\sum_{i=-\infty}^{\infty} \operatorname{sinc}^{\prime 2}(v)=\pi^{2} / 3$ for any real value of $v$ [22] and $\operatorname{sinc}^{\prime}(0)=0$, we have simplified (A11) as

$$
E\left\{r_{2,1}^{\prime 2}(D)\right\} \approx \frac{\pi^{2} N\left(\sigma_{s}^{2} \sigma_{q_{1}}^{2}+\sigma_{s}^{2} \sigma_{q_{2}}^{2}+\sigma_{q_{1}}^{2} \sigma_{q_{2}}^{2}\right)}{3} .
$$

Substituting (A8) and (A12) into (A10) with $\sigma_{q}^{2}=\sigma_{q_{1}}^{2}=\sigma_{q_{2}}^{2}$ and $\mathrm{SNR}=\sigma_{s}^{2} / \sigma_{q}^{2}$ yields (5).

Following the variance development for (A10), the covariance between $\hat{D}_{i, j}$ and $\hat{D}_{k, l}$ is then

$$
\begin{aligned}
& \operatorname{cov}\left(\hat{D}_{i, j}, \hat{D}_{k, l}\right) \\
& \approx E\left\{\left(\hat{D}_{i, j}-D_{i, j}\right)\left(\hat{D}_{k, l}-D_{k, l}\right)\right\} \\
& \left.\approx \frac{E\left\{r_{i, j}^{\prime}\left(\tilde{D}_{i, j}\right) r_{k, l}^{\prime}\left(\tilde{D}_{k, l}\right)\right\}}{E\left\{r_{i, j}^{\prime \prime}\left(\tilde{D}_{i, j}\right)\right\} E\left\{r_{k, l}^{\prime \prime}\left(\tilde{D}_{k, l}\right)\right\}}\right|_{\tilde{D}_{i, j}=D_{i, j}, \tilde{D}_{k, l}=D_{k, l}} .
\end{aligned}
$$

With the use of (A8), it is easy to see that

$$
\left.E\left\{r_{i, j}^{\prime \prime}\left(\tilde{D}_{i, j}\right)\right\}\right|_{\tilde{D}_{i, j}=D_{i, j}}=\left.E\left\{r_{k, l}^{\prime \prime}\left(\tilde{D}_{k, l}\right)\right\}\right|_{\tilde{D}_{k, l}=D_{k, l}} \approx-\frac{N \sigma_{s}^{2} \pi^{2}}{3} .
$$

In a similar manner, the numerator of (A13) can be derived as

$$
\begin{gathered}
\left.E\left\{r_{i, j}^{\prime}\left(\tilde{D}_{i, j}\right) r_{k, l}^{\prime}\left(\tilde{D}_{k, l}\right)\right\}\right|_{\tilde{D}_{i, j}=D_{i, j}, \tilde{D}_{k, l}=D_{k, l}} \\
\approx\left\{\begin{array}{cl}
\frac{N \sigma_{q_{i}}^{2} \sigma_{q_{j}}^{2} \pi^{2}}{3}, & i=k \text { and } j \neq l \text { or } i \neq k \text { and } j=l \\
-\frac{N \sigma_{q_{i}}^{2} \sigma_{q_{j}}^{2} \pi^{2}}{3}, & i=l \text { and } j \neq k \text { or } i \neq l \text { and } j=k \\
0, & i \neq j \neq k \neq l .
\end{array}\right.
\end{gathered}
$$

Putting (A14), (A15) into (A13) with $\sigma_{q}^{2}=\sigma_{q_{i}}^{2}=\sigma_{q_{j}}^{2}$ yields (6). 


\section{APPENDIX B}

In this Appendix, (12) will be proved, namely, $\mathbf{H}^{T} \mathbf{C}_{\mathbf{f}}^{-1}$ is proportional to $\mathbf{H}^{T}$. First of all, $\mathbf{J}^{T} \mathbf{J} \mathbf{J}^{T}$ is evaluated as follows. Recall (8)

$$
\mathbf{J}=\left[\begin{array}{llll}
\mathbf{J}_{1}^{T} & \mathbf{J}_{2}^{T} & \cdots & \mathbf{J}_{L-1}^{T}
\end{array}\right]^{T}
$$

where $\mathbf{J}_{i}=\left[\begin{array}{ll}\mathbf{0}_{(L-i) \times(i-1)} & -\mathbf{1}_{L-i} \mathbf{I}_{L-i}\end{array}\right]$, we have

$$
\mathbf{J}_{i}^{T} \mathbf{J}_{i}=\left[\begin{array}{ccc}
\mathbf{0}_{(i-1) \times(i-1)} & \mathbf{0}_{(i-1) \times 1} & \mathbf{0}_{(i-1) \times(L-i)} \\
\mathbf{0}_{1 \times(i-1)} & L-i & -\mathbf{1}_{L-i}^{T} \\
\mathbf{0}_{(L-i) \times(i-1)} & -\mathbf{1}_{L-i} & \mathbf{I}_{L-i}
\end{array}\right] .
$$

Hence

$$
\begin{aligned}
& \mathbf{J}^{T} \mathbf{J}=\sum_{i=1}^{L-1} \mathbf{J}_{i}^{T} \mathbf{J}_{i} \\
& =\left[\begin{array}{cccccc}
L-1 & -1 & -1 & -1 & \cdots & -1 \\
-1 & 1 & 0 & 0 & \cdots & 0 \\
-1 & 0 & 1 & 0 & \cdots & 0 \\
-1 & 0 & 0 & 1 & \cdots & 0 \\
\vdots & \vdots & \vdots & \vdots & \ddots & \vdots \\
-1 & 0 & 0 & 0 & \cdots & 1
\end{array}\right] \\
& +\left[\begin{array}{cccccc}
0 & 0 & 0 & 0 & \cdots & 0 \\
0 & L-2 & -1 & -1 & \cdots & -1 \\
0 & -1 & 1 & 0 & \cdots & 0 \\
0 & -1 & 0 & 1 & \cdots & 0 \\
\vdots & \vdots & \vdots & \vdots & \ddots & \vdots \\
0 & -1 & 0 & 0 & \cdots & 1
\end{array}\right] \\
& +\left[\begin{array}{cccccc}
0 & 0 & 0 & 0 & \cdots & 0 \\
0 & 0 & 0 & 0 & \cdots & 0 \\
0 & 0 & L-3 & -1 & \cdots & -1 \\
0 & 0 & -1 & 1 & \cdots & 0 \\
\vdots & \vdots & \vdots & \vdots & \ddots & \vdots \\
0 & 0 & -1 & 0 & \cdots & 1
\end{array}\right]+\cdots \\
& +\left[\begin{array}{cccccc}
0 & 0 & 0 & 0 & \cdots & 0 \\
0 & 0 & 0 & 0 & \cdots & 0 \\
0 & 0 & 0 & 0 & \cdots & 0 \\
\vdots & \vdots & \vdots & \vdots & \ddots & \vdots \\
0 & 0 & \cdots & 0 & 1 & -1 \\
0 & 0 & \cdot & 0 & -1 & 1
\end{array}\right] \\
& =L \mathbf{I}_{L}-\mathbf{1}_{L} \mathbf{1}_{L}^{T} \text {. }
\end{aligned}
$$

From (B2), we get

$$
\mathbf{J}^{T} \mathbf{J} \mathbf{J}^{T}=\left(L \mathbf{I}_{L}-\mathbf{1}_{L} \mathbf{1}_{L}^{T}\right) \mathbf{J}^{T}=L \mathbf{J}^{T}
$$

since $\mathbf{1}_{L} \mathbf{1}_{L}^{T} \mathbf{J}^{T}=\mathbf{0}_{L \times(L(L-1) / 2)}$.

Using (11) and (B3), $\mathbf{H}^{T} \mathbf{C}_{\mathbf{f}}^{-1}$ is then evaluated as

$$
\begin{aligned}
\mathbf{H}^{T} \mathbf{C}_{\mathbf{f}}^{-1} & =\mathbf{K}^{T} \mathbf{J}^{T} \mathbf{C}_{\mathbf{f}}^{-1} \\
& =-\frac{\pi^{2} N \mathrm{SNR}^{3}}{3(1+L \mathrm{SNR})}\left(L \mathbf{K}^{T} \mathbf{J}^{T}-\mathbf{K}^{T} \mathbf{J}^{T} \frac{1+L \mathrm{SNR}}{\mathrm{SNR}}\right) \\
& =\frac{\pi^{2} N \mathrm{SNR}{ }^{2}}{3(1+L \mathrm{SNR})} \mathbf{H}^{T}
\end{aligned}
$$

which is (12).

\section{APPENDIX C}

In this Appendix, (14), (16), and (17) will be proved. Using (B2), $\left(\mathbf{H}^{T} \mathbf{H}\right)^{-1}$ becomes

$$
\begin{aligned}
\left(\mathbf{H}^{T} \mathbf{H}\right)^{-1} & =\left(\mathbf{K}^{T} \mathbf{J}^{T} \mathbf{J} \mathbf{K}\right)^{-1} \\
& =\left(\mathbf{K}^{T}\left(L \mathbf{I}_{L}-\mathbf{1}_{L} \mathbf{1}_{L}^{T}\right) \mathbf{K}\right)^{-1} \\
& =\left(L \mathbf{I}_{L-1}-\mathbf{1}_{L-1} \mathbf{1}_{L-1}^{T}\right)^{-1}
\end{aligned}
$$

With the use of (C1), taking the inverse of (16) yields (17).

Using the matrix inversion lemma, (C1) can also be expressed as

$$
\begin{aligned}
& \left(L \mathbf{I}_{L-1}-\mathbf{1}_{L-1} \mathbf{1}_{L-1}^{T}\right)^{-1} \\
& =-\left(-L \mathbf{I}_{L-1}+\mathbf{1}_{L-1} \mathbf{1}_{L-1}^{T}\right)^{-1} \\
& =\left(L \mathbf{I}_{L-1}\right)^{-1}+\frac{\left(-L \mathbf{I}_{L-1}\right)^{-1} \mathbf{1}_{L-1} \mathbf{1}_{L-1}^{T}\left(-L \mathbf{I}_{L-1}\right)^{-1}}{1+\mathbf{1}_{L-1}^{T}\left(-L \mathbf{I}_{L-1}\right)^{-1} \mathbf{1}_{L-1}} \\
& =\frac{1}{L}\left(\mathbf{I}_{L-1}+\mathbf{1}_{L-1} \mathbf{1}_{L-1}^{T}\right) .
\end{aligned}
$$

From (12) and (C2), we easily obtain (16). Using (C2), $\left(\mathbf{H}^{T} \mathbf{H}\right)^{-1} \mathbf{H}^{T}$ is evaluated as follows:

$$
\begin{aligned}
& \left(\mathbf{H}^{T} \mathbf{H}\right)^{-1} \mathbf{H}^{T} \\
& =\frac{1}{L}\left(\mathbf{H}^{T}+\mathbf{1}_{L-1} \mathbf{1}_{L-1}^{T}\right. \\
& \left.\times\left[\begin{array}{lllll}
\mathbf{K}^{T} \mathbf{J}_{1}^{T} & \mathbf{K}^{T} \mathbf{J}_{2}^{T} & \mathbf{K}^{T} \mathbf{J}_{3}^{T} & \cdots & \mathbf{K}^{T} \mathbf{J}_{L-1}^{T}
\end{array}\right]\right) \\
& =\frac{1}{L}\left(\mathbf{H}^{T}+\mathbf{1}_{L-1} \mathbf{1}_{L-1}^{T}\right. \\
& \left.\times\left[\begin{array}{lllll}
\mathbf{I}_{L-1} & \mathbf{K}^{T} \mathbf{J}_{2}^{T} & \mathbf{K}^{T} \mathbf{J}_{3}^{T} & \cdots & \mathbf{K}^{T} \mathbf{J}_{L-1}^{T}
\end{array}\right]\right) \\
& =\frac{1}{L}\left(\mathbf{H}^{T}+\mathbf{1}_{L-1}\right. \\
& \left.\times\left[\begin{array}{lllll}
\mathbf{1}_{L-1}^{T} & \mathbf{0}_{(L-2) \times 1}^{T} & \mathbf{0}_{(L-3) \times 1}^{T} & \cdots & \mathbf{0}_{1 \times 1}^{T}
\end{array}\right]\right) \\
& =\frac{1}{L}\left[\begin{array}{ll}
\mathbf{I}_{L-1}+\mathbf{1}_{L-1} \mathbf{1}_{L-1}^{T} & \breve{\mathbf{H}}^{T}
\end{array}\right] .
\end{aligned}
$$

\section{APPENDIX D}

In this Appendix, we will prove (23). We first write $\mathbf{G}_{\mathbf{s}}$ as

$$
\mathbf{G}_{\mathbf{s}}=\mathbf{G}^{T} \mathbf{J}_{1}^{T}
$$

By using (B1), we have

$$
\mathbf{J}_{1}^{T} \mathbf{J}_{1}=\left[\begin{array}{cc}
L-1 & -\mathbf{1}_{L-1}^{T} \\
-\mathbf{1}_{L-1} & \mathbf{I}_{L-1}
\end{array}\right]
$$

Moreover, by the property of $\mathbf{J}$

$$
\begin{aligned}
\mathbf{J}_{1}^{T} \mathbf{1}_{L-1} & =\left[\begin{array}{c}
-L+1 \\
\mathbf{1}_{L-1}
\end{array}\right] \\
\Rightarrow \mathbf{J}_{1}^{T} \mathbf{1}_{L-1} \mathbf{1}_{L-1}^{T} \mathbf{J}_{1} & =\left[\begin{array}{cc}
(1-L)^{2} & (1-L) \mathbf{1}_{L-1}^{T} \\
(1-L) \mathbf{1}_{L-1} & \mathbf{1}_{L-1} \mathbf{1}_{L-1}^{T}
\end{array}\right]
\end{aligned}
$$


$\mathbf{G}_{\mathbf{s}} \mathbf{C}_{\mathbf{s}}^{-1} \mathbf{G}_{\mathbf{s}}^{T}$ is derived with the use of (C1), (D1) and (D2) as follows, which is (23):

$$
\begin{aligned}
\mathbf{G}_{s} \mathbf{C}_{s}^{-1} \mathbf{G}_{s}^{T}= & \frac{\pi^{2} N \mathrm{SNR}^{2}}{3(1+L \mathrm{SNR})} \mathbf{G}^{T} \mathbf{J}_{1}^{T} \\
& \times\left(L \mathbf{I}_{L-1}-\mathbf{1}_{L-1} \mathbf{1}_{L-1}^{T}\right) \mathbf{J}_{1} \mathbf{G} \\
= & \frac{\pi^{2} N \mathrm{SNR}^{2}}{3(1+L \mathrm{SNR})} \mathbf{G}^{T} \\
& \times\left[\begin{array}{cc}
L-1 & -\mathbf{1}_{L-1}^{T} \\
-\mathbf{1}_{L-1} & L \mathbf{I}_{L-1}-\mathbf{1}_{L-1} \mathbf{1}_{L-1}^{T}
\end{array}\right] \mathbf{G} \\
= & \frac{\pi^{2} N \mathrm{SNR}^{2}}{3(1+L S N R)} \mathbf{G}^{T}\left(L \mathbf{I}_{L}-\mathbf{1}_{L} \mathbf{1}_{L}^{T}\right) \mathbf{G} .
\end{aligned}
$$

\section{ACKNOWLEDGMENT}

The authors would like to thank the anonymous reviewers for their careful reading and constructive comments, which improved the clarity of this paper.

\section{REFERENCES}

[1] G. C. Carter, IEEE Trans. Acoust., Speech, Signal Process. (Special Issue on Time Delay Estimation), vol. 29, no. 3, Jun. 1981.

[2] G. C. Carter, Coherence and Time Delay Estimation: An Applied Tutorial for Research, Development, Test, and Evaluation Engineers. New York: IEEE Press, 1993.

[3] J. Chen, Y. Huang, and J. Benesty, "Time delay estimation," in Audio Signal Processing for Next-Generation Multimedia Communication Systems, Y. Huang and J. Benesty, Eds. Norwell, MA: Kluwer, 2004 , ch. 8

[4] Y. Huang, J. Benesty, G. W. Elko, and R. M. Mersereau, "Real-time passive source localization: A practical linear-correction least-squares approach," IEEE Trans. Speech, Audio Process., vol. 9, pp. 943-956, Nov. 2001.

[5] Y. Zhao, "Standardization of mobile phone positioning for $3 \mathrm{G}$ systems," IEEE Commun. Mag., vol. 40, no. 7, pp. 108-116, Jul. 2002.

[6] R. Yamasaki, A. Ogino, T. Tamaki, T. Uta, N. Matsuzawa, and T. Kato, "TDOA location system for IEEE 802.11b WLAN," in Proc. WCNC 2005, 2005, pp. 2338-2343.

[7] N. Patwari, J. N. Ash, S. Kyperountas, A. O. Hero, III, R. L. Moses, and N. S. Correal, "Locating the nodes: Cooperative localization in wireless sensor networks," IEEE Signal Process. Mag., vol. 22, no. 4, pp. 54-69, Jul. 2005.
[8] B. Yang and J. Scheuing, "A theoretical analysis of 2-D sensor arrays for TDOA based localization," in Proc. Int. Conf. Acoustics, Speech, Signal Process. (ICASSP), Toulouse, France, May 2006, vol. IV, pp. 901-904.

[9] B. Friedlander, "A passive localization algorithm and its accuracy analysis," IEEE J. Ocean. Eng., vol. 12, no. 1, pp. 234-245, Jan. 1987.

[10] J. O. Smith and J. S. Abel, "Closed-form least-squares source location estimation from range-difference measurements," IEEE Trans. Acoust., Speech, Signal Process., vol. 35, pp. 1661-1669, Dec. 1987.

[11] Y. T. Chan and K. C. Ho, "A simple and efficient estimator for hyperbolic location," IEEE Trans. Signal Process., vol. 42, no. 8, pp. 1905-1915, Aug. 1994.

[12] R. O. Schmidt, "A new approach to geometry of range difference location," IEEE Trans. Aerosp. Electron. Syst., vol. AES-8, pp. 821-835, Nov. 1972.

[13] R. O. Schmidt, "Least squares range difference location," IEEE Trans. Aerosp. Electron. Syst., vol. 32, no. 1, pp. 234-242, Jan. 1996.

[14] W. R. Hahn and S. A. Tretter, "Optimum processing for delay-vector estimation in passive signal arrays," IEEE Trans. Inf. Theory, vol. 19, pp. 608-614, Sep. 1973.

[15] C. H. Knapp and G. C. Carter, "The generalized correlation method for estimation of time delay," IEEE Trans. Acoust., Speech, Signal Process., vol. ASSP-24, no. 4, pp. 320-327, Aug. 1976.

[16] Y. T. Chan, J. M. F. Riley, and J. B. Plant, "Modeling of time-delay and its application to estimation of nonstationary delays," IEEE Trans. Acoust., Speech, Signal Process., vol. ASSP-29, no. 3, pp. 577-581, Jun. 1981.

[17] A. H. Quazi, "An overview on the time delay estimate in active and passive system for target localization," IEEE Trans. Acoust., Speech, Signal Process., vol. ASSP-29, no. 3, pp. 527-533, Jun. 1981.

[18] G. Jacovitti and G. Scarano, "Discrete time techniques for time delay estimation," IEEE Trans. Signal Process., vol. 41, no. 2, pp. 525-533, Feb. 1993.

[19] G. C. Carter, "Time delay estimation for passive sonar signal processing," IEEE Trans. Acoust., Speech, Signal Process., vol. 29, no. 3, pp. 463-470, Jun. 1981.

[20] B. Yang and J. Scheuing, "Cramér-Rao bound and optimum sensor array for source localization from time differences of arrival," in Proc. Int. Conf. Acoustics, Speech, Signal Processing (ICASSP), Philadelphia, PA, Mar. 2005, vol. IV, pp. 961-964.

[21] V. H. MacDonald and P. M. Schultheiss, "Optimum passive bearing estimation in a spatially incoherent noise environment," J. Acoust. Soc. Amer., vol. 46, no. 1, pt. 1, pp. 37-43, Jul. 1969.

[22] H. C. So, P. C. Ching, and Y. T. Chan, "A new algorithm for explicit adaptation of time delay," IEEE Trans. Signal Process., vol. 42, no. 7, pp. 1816-1820, Jul. 1994.

[23] J. P. Ianniello, "Large and small error performance limits for multipath time delay estimation," IEEE Trans. Acoust., Speech, Signal Process., vol. 34, no. 2, pp. 245-251, Apr. 1986. 